Hygiene for allowing us to make this comment in advance of publication.

-We are, etc.,

G. R. SMITH

Caroline J. Moryson

Nuffield Institute of Comparative Medicine,

The Zoological Society of London,
London N.W.1

1 Burns, G. F., and Williams, H., fournal of Hygiene, 1975, 74, 1.

W.H.O. Chronicle, 1975, 29, 55

of Hygiene. In press.

\section{Screening Methods for Covert Bacteriuria}

SIR,-As Dr. Rosalind Maskell (28 June, p. 752 ) and Mr. Roger Hole (19 July, p. 160) have indicated, the use of a single-medium dip-slide would undoubtedly simplify the dip-stream technique for domiciliary use by general practitioners and in population screening. Of the $1.4 \%$ spoilt dip-slides in our recent survey ${ }^{1}$ several showed culture media which had been fingered, possibly while attempting to rotate the dip-slide during micturition, in order to wet both sides.

In commenting on the choice of culture medium, Dr. Maskell's statement that "Use of the MacConkey surface, therefore, results in a number of false positive interpretations" misinterprets our results. It was the CLED medium which gave rise to the high false positive rate of $13.5 \%$ and, though this can be reduced to $1.8 \%$ by disregarding nonpathogenic organisms, to do so requires the discriminatory expertise of a microbiologist, generally implying a highly paid consultant. The method advocated by Mr. Hole takes into account our observation that the MacConkey medium suppressed some twothirds of the vulvovaginal contaminants, thus lowering the false positive rate to an acceptable $4.6 \%$ without giving rise to false negative results. This has the considerable attraction that large numbers of dip-slides could be interpreted inexpensively by a technician, and it must surely be regarded as the preferable method for population screening.

In hospital practice we are mainly concerned with confirmation of the diagnosis of urinary tract infection (U.T.I.), evaluation of treatment, and detection of asymptomatic relapses. The procedure employed in my renal clinic ${ }^{2}$ necessitates the collection of a midstream urine specimen for microscopical examination by the clinician and eliminates the need for culture in about $90 \%$ of samples. Since for this purpose the conventional immersion technique is used a singlemedium dip-slide at the same price as the double-coated version offers no advantage. I would therefore advocate the use of the standard dip-slide in hospitals and a MacConkey-only version for population screening.

In general practice the greatest problem of diagnosis arises through the multiplication of contaminant organisms when a delay of more than about an hour occurs between voiding urine in the surgery and its arrival at the local laboratory. A report of a colony count greater than $10^{8} / 1$ under these circumstances, of which neither the general practitioner nor the microbiologist may be aware can be seriously misleading. Since a high proportion of children with urinary tract infection have urinary tract abnormalities an I.V.P. should be regarded as mandatory in every confirmed case; an incorrect diagnosis of infection may therefore result in unnecessary exposure to radiation. Use of the dip-slide could completely eliminate this problem and it is therefore regrettable that its great potential in general practice does not seem to have received the widespread recognition which it deserves.-I am, etc.,

The Children's Hospital,

R. H. R. WHITE rmingham

Edwards, B., et al., British Medical fournal, 1975,

2 Robins, D. G., et al., Lancet, 1975, 1, 476.

London W.2

\section{Ulceration of Small Intestine and}

SIR,-I was interested to read the letter from Mr. S. J. Heffernan and Mr. J. J. Murphy (28 June, p. 746) recording small-intestinal ulceration and stricture associated with Slow-K tablets.

I have seen two examples recently of slowrelease potassium causing patients to present with perforation proximal to such strictures where the potassium was in fact contained in a mixed diuretic pill. One of these patients presented twice in two years with similar episodes before HydroSaluric-K was withdrawn from her hypertension management.

A 63-year-old woman was admitted in April 1973 witis what was thought to be spontaneous small-bowel perforation, and after oversewing of this she made a satisfactory recovery. Histological examination of the small bowel adjacent to the perforation was unhelpful. She was discharged from hospital still taking HydroSaluric-K one tablet each morning to control her hypertension and also methyldopa $500 \mathrm{mg}$, Saudo-K two tablets, and clonidine $100 \mu \mathrm{g}$, each thrice daily.

She presented again this year with acute abdominal pain of sudden onset and clinical evidence of peritonitis. At laparotomy a further smallbowel perforation proximal to a stricture was identified. It seemed highly likely that the slowrelease potassium contained in HydroSaluric-K was responsible for her stricture and subsequent perforation on both occasions.

The combination of slow-release potassium with other drugs in this case probably allowed it to go unnoticed as the causative agent, and it is worth remembering that the small bowel cannot distinguish between Slow-K tablets and slow-release potassium included in other medications. - I am, etc.,

\section{Kent and Canterbury Hospital,}

TOM TREASURE

Canterbury

\section{Doctor's Rights}

SIR,-As one who has suffered from the attentions of the Health Service Commissioner I deprecate the complacency shown in your leading article (28 June, p. 710). Your readers should realize that the commissioner and his agents are armed with the widest powers to compel attendance and extract statements and inspect confidential clinical records. How much do these men know about the clinical process and the im portance of the relation between patient and doctor? They do not hesitate to question clinicians on clinical matters and to pronounce judgement on such matters. Your readers should know too that the commissioner's activities are already encroaching on the doctor's right of independent judgement and, in my view, harming the relation between patients and doctors and damaging the interests of patients in particular and of the Health Service in general.

I hope, and many others doubtless share this hope, that the B.M.A. will take tine opportunity offered by the presentation of the Health Service Commissioner's first report to appoint a working party to examine that report and to comment on the methods used and the opinions expressed.-I am, etc.

GEORGE BONNEY

SIR,-According to your leading article (28 June, p. 710) the crucial factor in any doctorpatient relationship is mutual trust and therefore at any point either party can withdraw without giving any reason simply as a fundamental right. However, if a doctor has agreed to treat a patient surely he is still under an obligation to ensure that the patient is transferred to the care of another colleague so that he does not suffer as a result of the doctor's decision. Doctor and patient do not stand on equal terms in this unwritten contract and the patient is the more vulnerable of the two. Surely this aspect of professional freedom should be more thoroughly examined. Perhaps it is true that patients do not complain precisely because they fear personal retribution and it is not a sign of maturity to cover the issue with a cloak of dictatorial dogma.-I am, etc.,

Stockton Heath

M. D. THOMAS

Warrington, Cheshire

\section{Appointment of Consultants and}

\section{Termination of Pregnancy}

SIR,-The memorandum issued by the Chief Medical Officer in February referring to "Appointment of Consultants: Termination of Pregnancy" makes it clear that in certain areas (presumably those covered by only one consultant gynaecologist), those opposed to such termination will not be appointed. One can sympathize with the Ministerial problem -they have an Abortion Act to put into practice and the N.H.S. has been saddled with it. But there is a subtle danger in this: a number of areas (how many I do not know) will inevitably come to be manned only by proabortion doctors. There is nothing to compel any woman to have an abortion, but on the other hand every G.P. knows that the pressure to have an abortion does not always come from the woman. Those women who prefer to be attended by doctors who value life in all stages are being denied the exercise of this choice. Moreover, the lack of job availability will inevitably mean a gradual disappearance from certain branches of the medical profession of those doctors who value human life dearly-what a reflection on our present-day society!

The medical profession nowadays is widely split on matters of ethics, whereas once there was unity. If in the eyes of our patients we come to be seen only as technicians we shall deserve to lose that respect gained for our profession by our predecessors. Whatever their therapeutic limitations, they were for the most part seen as compassionate men, striving always to preserve and save life, and 